\title{
Les PME multimédia et logiciel éditeur dans le pôle de compétitivité « Solutions Communicantes Sécurisées »: Quel mode de gouvernance pour quelle intégration industrielle?
}

\section{Multimedia and software SMEs in the competitive cluster « Solutions Communicantes Sécurisées » : Which governance model for industrial integration?}

\author{
Martine Gadille \\ Chargée de recherche \\ Laboratoire : LEST UMR 6123 Université Aix-Marseille/CNRS
}

\begin{abstract}
Maud Pélissier
Maître de Conférences Laboratoire i3M, Université du Sud Toulon-Var,
\end{abstract}

Résumé Cet article traite de l'intégration industrielle de PME se situant en marge des projets de $R \& D$ d'un pôle alors même qu'elles représentent un enjeu de développement économique pour le territoire. Nous mobilisons la notion de non-complémentarité des connaissances pour éclairer cette situation et établir un diagnostic sur un plan technologique. Nous montrons qu'une issue favorable pour sortir de cette difficulté ne peut être envisagée qu'à l'échelle d'une gouvernance territoriale unissant l'ensemble des acteurs territoriaux de l'innovation.

Mots clés Intégration industrielle, PME, pôle de compétitivité, Solutions Communicantes sécurisées, gouvernance territoriale

\begin{abstract}
This article deals as the not present industrial integration of SMEs in the $\mathrm{R} \& \mathrm{D}$ projects of a cluster even when they are at the stake in the economic development policy of the Region. We mobilize the notion of knowledge fungeability to enlighten this situation and we show that collective learning can be achieved on the basis of a territorial governance of innovation enhancing this integration.
\end{abstract}

\begin{tabular}{lllll}
\hline Key words & $\begin{array}{l}\text { Industrial integration, } \\
\text { Communicantes sécurisées, territorial governance }\end{array}$ & cluster, & Solutions \\
\end{tabular}


Martine Gadille est chargée de recherche en économie et gestion au LEST-UMR CNRS 6123. Ses thèmes de recherche portent principalement sur la gestion du changement et la théorie des organisations (appropriation des TIC dans les PME et gouvernance régionale de l'innovation). Elle enseigne en master recherche en management des connaissances et de l'innovation. Elle a récemment coordonné une recherche-action auprès d'un pôle de compétitivité sur la question de l'évolution des pratiques de gestion des ressources humaines et des compétences dans les PME confrontées à des tensions sur le marché du travail dans un contexte stratégique d'innovation et de diversification des marchés.

Maud Pélissier est maître de conférences en sciences économiques à l'université Jean Monnet de Saint Etienne et membre de l'équipe de recherche du CREUSET ( CNRS FRE 2938). Ses thèmes de recherche portent sur l'économie des réseaux et l'économie de l'innovation, le développement local et l'intelligence économique. Elle est actuellement responsable pédagogique d'un Master spécialisé dans l'ingénierie du multimédia à l'université de Toulon. A ce titre, ses enseignements principaux portent sur l'intelligence territoriale et l'économie du multimédia. 


\section{Introduction}

L'un des enjeux majeurs posés à de nombreux pôles de compétitivité réside dans leur capacité à créer et à encourager, à l'échelle du territoire, des liens de proximité organisationnelle ${ }^{4}$ entre des entreprises qui n'ont pas coutume de coopérer autour de projets d'innovation. Tel est le cas du pôle de compétitivité, que nous proposons d'étudier, dénommé "Solutions Communicantes Sécurisées », localisé dans la région Provence Alpes Côte d'Azur et spécialisé dans le domaine des technologies de l'information et de la communication.

Lors de la création de ce pôle, le discours officiel des instances fondatrices véhiculait un objectif ambitieux puisqu'il proposait, dans un contexte technologique de convergence numérique, de mettre en relation un spectre très large d'activités industrielles allant « du silicium aux usages ». Il prônait en particulier un modèle de coopération inter-entreprises dépassant les frontières sectorielles traditionnelles et conduisant à un rapprochement entre les industries de la microélectronique, des télécommunications, du logiciel et enfin du multimédia.

L'objet de notre papier porte sur les freins et la dynamique de cette coopération du point de vue des savoirs en jeux et de la régulation sociale à l'œuvre. Ce travail permet d'introduire des pistes d'action envisageables, à partir de l'expérience, pour remédier aux difficultés rencontrées.

Dans cette perspective, nous avons choisi de focaliser notre attention sur des PME du «logiciel éditeur » et du «multimédia de contenu ». Celles-ci, bien que présentes dans le pôle - soit directement en tant que membre à part entière, soit indirectement par l'intermédiaire de leurs associations professionnelles respectives - semblent en même temps les plus éloignées, en terme de «systèmes technologiques » (Carlsson, 1994) et de spécialisation des savoirs (Antonelli 2003), du paradigme technologique dominant du pôle (la microélectronique).

Ce constat nous semble important à souligner car il peut être le signe annonciateur de l'exclusion progressive de ces PME du pôle. Diagnostiquer et remédier à ce risque probable apparaît important au vu du dynamisme croissant des marchés sur lequel se situent ces entreprises à l'échelle locale comme au niveau international.

L'enjeu soulevé ici est celui des apprentissages collectifs et de l'action collective (Reynaud, 1997) susceptibles d'être initiés sur un même territoire, entre des «systèmes technologiques » qui, bien qu'éloignés à court terme, pourraient s'avérer complémentaires à moyen/long terme. Nous voudrions ainsi montrer que face à l'ampleur d'un tel enjeu, le pôle ne peut y remédier seul, et qu'il a tout intérêt à participer à une gouvernance territoriale plus à même d'assurer, au niveau régional, non seulement la coordination, mais aussi la régulation sociale, au sein et entre les différentes « communautés professionnelles » ${ }^{5}$ impliquées.

La méthode mobilisée repose sur des enquêtes de terrain effectuées sous forme d'entretiens semi-directifs et d'observation participante dans le cadre des associations professionnelles de PME et de réunions du pôle.

Après avoir présenté le socle théorique de notre étude ainsi que sa méthodologie, nous exposons l'étude de cas pour en tirer enfin des enseignements en matière de gouvernance

\footnotetext{
${ }^{4}$ Nous faisons référence à la définition de Rallet et Torre : «by organized proximity, the ability of an organization to make its members interact is meant. The organization facilitates interactions within it and makes them a priori easier than with units situated outside the organization $\gg(2005$, p. 49).

${ }^{5}$ La définition retenue ici par Dibiaggio et Ferrary (2003) est à rapprocher de la définition d'une clique dans l'approche internationale en terme de réseaux sociaux : c'est un groupe social d'individus reliés entre eux par des liens (de production de savoir) forts.
} 
territoriale et d'apprentissages collectifs dépassant les frontières organisationnelles du pôle au sens strict.

\section{L'intégration d'activités industrielles dans un pôle de compétitivité}

Le processus d'intégration d'acteurs économiques hétérogènes dans un pôle de compétitivité peut être analysé selon deux axes théoriques complémentaires. Le premier axe est constitué par l'approche des systèmes technologiques d'innovation mettant l'accent sur la nature et la localisation des connaissances technologiques, comme facteurs déterminants dans une dynamique locale d'innovation. Le second axe traite de la notion de gouvernance territoriale comme catégorie pertinente pour analyser les apprentissages liés à la construction de l'espace légitime d'intervention de la structure d'un pôle.

\subsection{Les Systèmes technologiques et l'hétérogénéité des connaissances dans l'innovation}

Une des difficultés principales du travail de coordination dans le dispositif des pôles de compétitivité est de mettre en ouvre des moyens facilitant les collaborations non seulement entre des concurrents au sein d'un même secteur mais aussi entre des populations d'entreprises aux bases technologiques et sectorielles différentes et potentiellement complémentaires. A notre sens, la question de la confiance n'est pas première pour expliquer une telle difficulté. Le cadre théorique qui permet, en premier lieu, d'éclairer cette difficulté, a été élaboré, dans une perspective évolutionniste, par des analystes qui ont exploré la nature cumulative et dynamique $\mathrm{du}$ changement technique. La notion de «trajectoire technologique » dégagée de ces travaux permet de souligner que la direction du changement technologique est contrainte par les avancées antérieures, la nature cumulative des connaissances, les normes et les croyances (Pavitt, 1984). La notion complémentaire de «paradigme technologique » sert à qualifier le chemin de dépendance dans lequel s'inscrivent les acteurs porteurs du changement technologique. Il se définit par l'existence d'une vision, d'un ensemble de procédures, d'une définition des problèmes pertinents et de connaissances spécifiques liées à leur résolution (Dosi, 1982). Dans un tel cadre, on comprend que l'exercice de coordination entre des activités complémentaires inscrites dans différents paradigmes est une entreprise qui doit permettre de déplacer les savoirs, les croyances, représentations de différentes communautés d'acteurs qui les constituent.

Dans cette même perspective, Carlsson (1994) suggère que la prise en compte des barrières liées aux connaissances technologiques importe plus que celles des frontières nationales ou régionales pour expliquer les stratégies de partenariat et d'implantation des firmes dans le changement industriel. L'intérêt de la notion de systèmes technologiques (ST) qu'il a développée est alors de saisir plus finement les enjeux liés à la formation des réseaux industriels où sont articulés et contrôlés des blocs de connaissances techniques. Carlsson et Stankiewicz (1995) définissent un ST comme un réseau d'agents interagissant dans chaque domaine technologique spécifique dans des contextes institutionnels donnés et dont les finalités sont la création, la diffusion et l'utilisation d'une technologie. Les ST peuvent être régionaux, locaux, nationaux ou internationaux dans la mesure où leurs frontières vont dépendre des circonstances technologiques, de marché et de l'interdépendance des acteurs et des institutions. En l'occurrence, c'est la création de ST au niveau de l'espace de coordination d'un pôle qui nous importe. Cette création implique l'établissement de passerelles en termes d'information et de savoirs entre différents paradigmes technologiques soutenant des ST déjà constitués et ancrés sous forme de groupes sociaux ou communautés professionnelles dans le territoire (Dibiaggio et Ferrary, 2003). 
Pour avancer sur cette piste de recherche, il nous semble opportun d'intégrer à ce niveau les travaux d'Antonelli (2003) qui identifient différents types de connaissances mobilisées dans le processus d'innovation. Pour cet auteur, la connaissance technologique nécessaire pour produire et diffuser l'innovation est caractérisée par un haut niveau de complexité. Parmi ces connaissances technologiques, certaines ne peuvent se combiner qu'à une variété réduite d'activités, de nouveaux produits ou processus. C'est cet aspect complémentaire qui fait leur valeur. D'autres blocs de connaissances technologiques, par contre, peuvent avoir un potentiel d'application beaucoup plus large. C'est ce qu'Antonelli désigne par la fongibilité ${ }^{6}$, définie par l'étendue et l'application de ces éléments de connaissance.

Selon cet auteur, les technologies de l'information et de la communication sont concernées par ces deux aspects. Elles résultent de complémentarités technologiques entre une grande variété de champs de connaissances (l'électronique, l'informatique, les télécommunications, etc.) et conduisent à l'émergence de nouveaux systèmes technologiques associés à la recombinaison de différents blocs de savoirs. En même temps, ces systèmes sont applicables à un ensemble très large d'activités économiques et humaines.

Deux principales propositions se dégagent de cette analyse. D'une part, la faible implication des PME dans les projets de R\&D d'un pôle peut être lue en fonction des aspects complémentaires ou fongibles de leurs connaissances vis-à-vis du paradigme technologique dominant. D'autre part, l'évolution de la spécialisation industrielle de différents territoires dans la production d'une nouvelle technologie apparaît liée à la localisation et la mise en relation de différents blocs de connaissances complémentaires ou fongibles, déplaçant la dynamique du paradigme technologique dominant. C'est à ce dernier niveau que la gouvernance territoriale prend tout son sens comme nous allons le suggérer dans la section suivante.

\subsection{Le renforcement d'une gouvernance territoriale et ses tensions}

En tant que système territorialisé d'innovation, la mission d'un pôle de compétitivité peut se définir en terme de «management organisationnel » dont les finalités essentielles peuvent être définies de la façon suivante : définir une stratégie globale, coordonner les acteurs et contrôler la cohésion du réseau (Elingher et al., 2007). Toutefois, cette perspective managériale pour le moins originale reste entièrement subordonnée à un objectif plus global de développement économique territorial (local). La question qui se pose alors est celle de l'articulation des actions du pôle avec celles initiées par d'autres acteurs territoriaux (publics et privés), tout autant légitimes en matière de développement économique local. Or, à ce niveau, force est de constater un enchevêtrement des légitimités qui peut risquer de nuire à l'efficacité de la dynamique d'innovation.

Tout d'abord, rappelons que, même si les structures de gouvernance des pôles de compétitivité sont autonomes dans leur feuille de route, elles demeurent un dispositif piloté ${ }^{7}$ ultimement à l'échelon national. C'est l'Etat centralisateur qui décide de l'évolution et du devenir de ce dispositif. Se pose donc la question de l'articulation de cette politique centralisée avec la politique d'innovation mise en œuvre à un niveau décentralisé par les Conseils Régionaux. Rappelons en effet qu'à l'ère de la glocalisation (Longhi et Spindler, 1999), la recherche de compétitivité au niveau local a conduit à un renforcement progressif des Conseils Régionaux comme acteurs incontournables du développement économique et de

\footnotetext{
${ }^{6}$ « Fungeability defines the dowstream complementarity of any bit of knowledge. Some elements of technological knowledge may apply to a narrow and specific range of activities, either new products or new processes. Fungeability is defined and measured by the scope of application of a new bit of knowledge » (2003, p. 598).

${ }^{7}$ Nous voulons signifier ici le fait que l'évolution des pôles de compétitivité se décide à l'échelon centralisé de l'Etat.
} 
l'innovation. Des tensions multiples sont ainsi susceptibles de naître au sein de cet espace complexe d'innovation que constitue le territoire à l'échelle régionale.

L'émergence des pôles a ainsi créé un espace de tension inévitable avec les acteurs déjà présents sur le territoire. Pour leur part, les pôles sont généralement pilotés par des acteurs privés, issus majoritairement des entreprises innovantes issues du territoire, qui n'accordent que peu de pouvoir décisionnel aux acteurs publics territoriaux. En particulier, l'institution politique régionale, qui s'est vue attribuer depuis les lois de décentralisation une autonomie croissante dans le «management » du développement local, n'a pas été directement associée à la mise en place des pôles de compétitivité à l'échelle de leur territoire. D'une manière générale, les collectivités territoriales jouent seulement un rôle de prescripteur de second plan ${ }^{8}$ dans la trajectoire d'innovation régionale conduite par les pôles.

Enfin, les structures de gouvernance des pôles sont aussi confrontées à des tensions internes au sens où leurs missions s'inscrivent dans une double dynamique globale et locale. Rappelons en effet qu'un pôle, - et de façon encore plus significative lorsqu'il a été labellisé «pôle mondial »- en raison de son potentiel à créer de fortes synergies en matière de R\&D et des produits nouveaux à forte valeur ajoutée, a pour vocation première de participer à la compétitivité et à l'attractivité de l'économie française. Ainsi, même s'il a vocation à soutenir le développement territorial, cela reste un objectif secondaire dans son plan d'action stratégique. De plus, rien ne garantit a priori que les actions de soutien destinées à créer des synergies de collaboration autour de projets de R\&D conduisent automatiquement à favoriser un tel développement et, en particulier, la compétitivité des filières industrielles sur lesquelles repose ce dernier.

L'intégration industrielle des PME dans un pôle de compétitivité sera dans de nombreux cas, accompagnée de façon optimale seulement si elle résulte d'une coordination entre la gouvernance opérationnelle (i.e. managériale) du pôle et l'ensemble des acteurs qui, sur le territoire, ont une légitimité d'action en terme d'innovation (Pélissier, 2008). L'enjeu se situe au niveau d'une gouvernance territoriale qui peut se définir comme «l'ensemble des processus institutionnels qui participent à la régulation sociale du système économique territorial» (Gilly et Perrat, 2004, p. 93). Il s'agit en effet dans le cas étudié ici de faire émerger par un jeu de compromis entre, d'une part, des rapports de pouvoir à l'échelle locale et, d'autre part, des tensions entre des régularités «verticales» de type sectoriel et macro institutionnel un mode de régulation collectif efficace. La question de l'implication des PME du logiciel éditeur et du multimédia dans les projets R\&D d'un pôle de compétitivité peut donc être comprise au regard des actions concertées entre la gouvernance du pôle, les représentants des différents systèmes techniques en jeu et les représentants de la Région (Conseil Régional et Etat en région).

\subsection{La méthode d'observation}

L'observation de terrain a été réalisée sur la période fin 2006- printemps 2008. Elle a été menée par le biais de trois méthodes principales afin de permettre une triangulation des données pour éviter les biais: l'entretien semi-directif, l'observation participante et la collecte de données secondaires issues en partie des acteurs observés. Elle intervient en complément de différentes monographies réalisées par les collègues au sein de l'équipe de recherche à laquelle nous nous sommes jointes (Garnier et Lanciano, 2008 ; Lanciano et Nohara, 2008) S'agissant de l'entretien semi-directif, la question ouverte principale a porté sur le «positionnement» de l'acteur interviewé vis-à-vis du pôle. Pour les responsables

\footnotetext{
${ }^{8}$ Elles peuvent en effet faire le choix de participer financièrement aux projets d'innovation et/ou au fonctionnement de la structure d'animation du pôle.
} 
d'entreprise, cette question a été précédée d'une demande de description de l'activité de l'entreprise, de sa technologie et de son historique de création. Pour les responsables d'association, il leur a été demandé de présenter l'historique et les raisons de la création de l'association.

Les entretiens semi-directifs et confidentiels ont porté sur différentes catégories de populations et ont permis également de trianguler les données récoltées. Ces différentes catégories sont les suivantes :

- Présidents ou collaborateurs proches, représentant chacune des sept associations professionnelles impliquées dans le pôle (7 personnes);

- Responsables d'entreprises adhérentes (ou non) dans le pôle, ayant participé (ou non) à des projets $\mathrm{R} \& \mathrm{D}$, répartis sur l'ensemble du territoire géographique du pôle et dont l'activité est incluse dans la définition de l'aire d'intervention du pôle (44 responsables) ;

- Responsables opérationnels du pôle : directeur général, directeur adjoint, coordonnateur projets $\mathrm{R} \& \mathrm{D}$, responsables coordination PME (4 personnes, dont les deux dernières rencontrées à deux reprises) ;

- Chargés de mission du Conseil Régional et leurs responsables (services recherche et service développement économique) : cinq personnes dont le responsable des dossiers du pôle rencontré de façon récurrente.

L'observation participante a été mise en œuvre auprès des associations du logiciel éditeur et du multimédia (une vingtaine de réunions d'adhérents et manifestations avec invités, entre 2006 et 2008) ainsi qu'auprès des groupes thématiques du pôle (sur l'année 2007, cinq réunions).

La collecte de documents a trois sources distinctes :

- Des documents concernant les associations adhérentes du pôle: projets d'actions collectives soumis au financement régional, comptes rendus de rencontres...

- Des documents produits par le pôle : informations issues de leur site web, des forums PME organisés par la cellule communication du pôle...

- Des documents fournis par les responsables de mission du Conseil Régional.

L'exploitation des données a été réalisée de façon manuelle, par l'analyse thématique avec recherche d'attitudes (comportement cognitifs et expression de rapports coopératifs ou antagoniques).

\section{Les trajectoires et systèmes technologiques du pôle SCS}

L'étude de cas présentée dans cette partie révèle tout d'abord l'importance de l'histoire industrielle régionale dans la genèse du pôle et dans l'émergence du paradigme technologique dominant qui lui est associé. Elle nous permet ensuite de mettre en évidence les différents blocs de connaissances non articulés lors de la création du pôle et présente enfin les déplacements effectués sur cette base en matière d'intégration industrielle des PME du logiciel éditeur et du multimédia dans le cadre de la gouvernance territoriale.

\subsection{La genèse historique du pôle SCS}

Le pôle SCS est né d'une volonté collective de rapprochement entre deux espaces productifs d'innovation, chacun appartenant à l'industrie des TIC (technologies de l'information et de la communication) mais chacun ayant connu une trajectoire autonome de développement territorial (Garnier, 2008). L'émergence de l'industrie des TIC en région PACA remonte au milieu des années 70 avec le développement progressif de ces deux espaces productifs géographiquement distants puisque le premier se trouve à l'ouest de la région, dans la zone de Rousset-Gémenos et le second à l'est, dans la technopole de Sophia Antipolis. A ce 
décentrement géographique fait écho une spécialisation technologique entre ces deux espaces. Dans la zone «Paca ouest », se sont localisées les grandes entreprises de la microélectronique (les fondeurs) comme Atmel, ST Microelectronics ou Dupont Photomask, puis ultérieurement l'entreprise leader dans la carte à puce, Gemplus, et enfin tout un ensemble d'entreprises créées par essaimage lors des périodes de crise dans les années 80-90. Très rapidement, il est apparu qu'une faiblesse de ce tissu, en terme d'ancrage territorial, était l'absence d'une proximité avec une structure locale de recherche, les entreprises présentes à l'origine ayant très majoritairement une seule vocation de production. Faiblesse car les entreprises de la microélectronique et, en particulier, les «fondeurs » sont, par essence, des entreprises très «nomades », dont les activités de production sont soumises à de fortes menaces de délocalisation. Il est rapidement apparu que la création de liens avec le monde de la recherche publique constituait une opportunité susceptible de favoriser l'ancrage de ce tissu productif. Ainsi, comme le souligne Zimmerman (1998), ce n'est qu'avec la création de Gemplus (en 1987) que se développe progressivement un début de milieu innovateur caractérisé par une proximité du pôle «carte à puce» avec l'aval utilisateur et quelques laboratoires de recherche ${ }^{9}$.

Dans la zone de Sophia Antipolis, l'émergence d'un espace productif spécialisé dans les TIC s'inscrit dans le cadre du développement de la Technopole. Progressivement sont venues s'implanter sur cette zone des grandes entreprises spécialisées dans la production de semi conducteurs (NXP, Texas Instrument et Infineon), des entreprises des télécommunications et du logiciel puis des laboratoires de recherche dépendant de l'Ecole des Mines de Paris et de l'INRIA.

Les acteurs économiques de ces deux espaces productifs ont été animés par la même volonté de structurer plus fortement leur tissu économique respectif en vue d'être plus armés face aux crises récurrentes auxquelles ils avaient dû faire face dans le passé. Les uns et les autres eurent alors le projet similaire de créer un véritable milieu innovateur pour pallier en particulier le déficit des relations science/industrie. Ce mouvement de structuration, résultant d'apprentissages collectifs, s'est traduit par la création d'associations professionnelles ${ }^{10}$ dont la vocation principale était de favoriser l'émergence d'un réseau territorialisé d'innovation sur ces deux espaces productifs. Précisons ici que l'ensemble de ces associations sont aujourd'hui membres du pôle SCS et un grand nombre d'entre elles ont le statut de membre fondateur.

Dans la dynamique de ces deux trajectoires territoriales, les collectivités territoriales (et notamment le Conseil Régional) n'ont cessé de jouer un rôle de premier plan. Dans une première phase, elles ont fortement appuyé et incité à la structuration respective de ces deux espaces productifs en participant financièrement aux projets collaboratifs d'innovation initiés dans le cadre de ces associations ${ }^{11}$. Dans une seconde phase, une modification de stratégies a été mise en place, oeuvrant dans le sens d'un nécessaire rapprochement entre ces deux espaces. Dès lors, comme le soulignent Lanciano et Nohara (2008), «les acteurs publics locaux ont conditionné leurs soutiens financiers à la construction d'un seul espace scientifique et technique régional. L'ensemble des intervenants se sont alors recentrés au début des années 2000 sur l'idée de créer une filière régionale de l'électronique allant du " silicium aux objets communicants » (2008, p. 78). Cela s'est traduit par la création d'un

\footnotetext{
${ }^{9}$ «Le seul argument qui rende capable de rivaliser avec ce nomadisme inhérent à l'activité est celui d'une dynamique d'innovation, fondée non pas seulement sur des activités de $R \& D$ à proprement parler, mais également sur une capacité à résoudre des problèmes effectifs posés par les spécifications des applications. Les exemples fourmillent qui montrent que la proximité avec l'aval utilisateur constitue une source d'efficacité stratégique » (1998, p. 49)

${ }^{10}$ Dans la zone Paca ouest, a été créée au départ l'association du CREMSI (Centre Régional d'Etude de la Microélectronique sur le Silicium) (1993). Dans la zone Paca Est, a été créée en 2004 l'association SAME (Sophia Antipolis Micro Electronics). Notons aussi la création d'autres associations reliées au tissu microélectronique : Telecom Valley (1991) sur Sophia Antipolis et Baby Smart (2005) dans la zone métropolitaine marseillaise.

${ }^{11}$ Cela a surtout été le cas pour l'association CREMSI
} 
Centre Intégré de Microélectronique (CIM Paca) en $2003^{12}$, dont la vocation était de permettre l'émergence d'un milieu innovateur performant à l'échelle régionale. L'originalité de ce centre est d'abriter la création de trois plate-formes technologiques, chacune offrant aux entreprises et laboratoires de recherche un lieu de mutualisation des moyens de recherche et de développement (ibid) ${ }^{13}$.

Ce changement de stratégie territoriale est important à mentionner car il prit forme au moment même où l'appel à projets sur les pôles de compétitivité fut lancé. Ainsi, pour les acteurs de la filière microélectronique, Cim Paca, dispositif de plate-formes géré par l'Association pour la Recherche sur les Composants et les Systèmes Intégrés Sécurisés (ARCSIS), constituait une candidature parfaitement adaptée au cahier des charges de cette politique nationale d'innovation. Toutefois, l'idée d'adouber du sceau «pôle de compétitivité » une structure déjà existante ne fit pas l'unanimité du côté des institutions politiques. Les négociations qui eurent lieu au niveau régional entre les différents protagonistes (grandes entreprises, pouvoirs publics locaux et préfecture de région) aboutirent ainsi à proposer un projet plus ambitieux en élargissant aux secteurs des télécommunications et du logiciel. Par la suite, les acteurs du Conseil Régional, avec l'accord des services de l'Etat, demandèrent d'intégrer les acteurs situés le plus en aval de la filière TIC présents en Paca, les éditeurs de logiciels applicatifs à destination des entreprises et les entreprises du multimédia producteurs de contenus numériques (cf. Figure 1).

\section{Figure 1 : La dynamique de convergence du Pôle SCS (Source : Pôle SCS)}

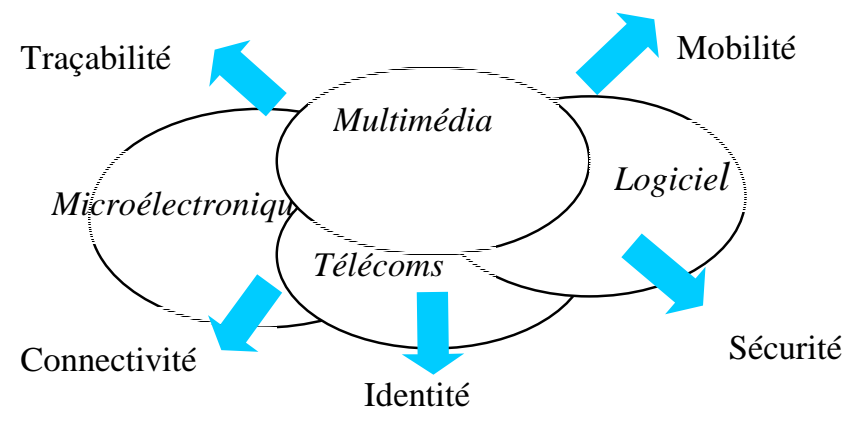

Ces derniers, constitués majoritairement de PME et TPE, commençaient à avoir une certaine visibilité notamment grâce à leurs associations professionnelles ${ }^{14}$ qui étaient très actives sur le territoire et vis-à-vis des pouvoirs publics locaux en particulier en matière de lobbying. C'est sous cette nouvelle forme que le pôle SCS a été labellisé. Son activité de mise en réseau se définissant dorénavant comme allant du «silicium aux usages » (et non plus seulement du «silicium aux objets communicants») unit ainsi 4 types d'activités industrielles: la microélectronique, les télécommunications, le logiciel et le multimédia.

En définitive, il nous semble important de souligner le caractère à la fois ambitieux mais périlleux de ce pôle de compétitivité. Ambitieux car il est vrai que sur un plan technologique, tous ces acteurs sont au cœur de la révolution du numérique et ainsi porteurs de dynamiques d'innovation caractérisées par un fort degré d'opportunités technologiques. Périlleux dans la

\footnotetext{
${ }^{12}$ Approuvé par une décision nationale du CIADT du 18 décembre 2003.

${ }^{13}$ Ces plate-formes «s'inscrivent dans une chaîne de création de valeur qui traverse le cœur de l'activité électronique » (Lanciano-Morandat et Nohara, ibid) et sont dédiées respectivement à la conception de systèmes électroniques, à la production de semi conducteurs et aux nouvelles applications des circuits intégrés. Les trois métiers de la microélectronique concernés sont ceux du logiciel (réseau et système) puis de la fonderie et du back end.

${ }^{14}$ Medinsoft pour les éditeurs de logiciels entreprise et Med multimédia pour les entreprises du multimédia.
} 
mesure où la structure décisionnelle du pôle a pour mission, du point de vue de la Région, d'établir des passerelles entre des acteurs issus de quatre systèmes technologiques différents et cloisonnés et de veiller à l'intégration des PME dans les projets R\&D. A ce titre, l'intégration industrielle des entreprises logiciels entreprise et multimédia va constituer un des enjeux de la gouvernance du pôle SCS.

\subsection{La complémentarité des connaissances des PME et leur intégration dans les projets du pôle}

Les chiffres présentés à intervalle régulier par le pôle SCS affichent un degré d'implication des PME dans les projets collaboratifs d'innovation plutôt satisfaisant (40\% des porteurs de projets sont des PME, 40\% des acteurs impliqués dans les projets sont des PME et 1/3 des financements des projets sont allés vers les PME) $)^{15}$. Toutefois, si l'on regarde de plus près, il apparaît de façon flagrante que ces mêmes chiffres dissimulent des contrastes importants au sein de la population des PME, certaines activités de PME étant quasiment absentes. Tel est le cas du multimédia de contenu (communication, ludo-éducatif, ...) et du logiciel éditeur spécialisé sur les système d'information d'entreprise (progiciels intégrés de gestion, ebusiness, ...).

Ce résultat peut être éclairé par une analyse en terme de complémentarité et fongibilité des connaissances. Ce dernier conduit à situer plus précisément les PME du logiciel éditeur et du multimédia. On observe ainsi la co-existence de trois groupes de PME réparties selon leur degré d'intégration vis-à-vis des briques de connaissances du cœur du paradigme technologique (cf . schéma 2).

Un premier groupe rassemble des entreprises au cœur du paradigme technologique dominant sur le territoire : celui de la microélectronique. Il s'agit notamment de start up positionnées sur des blocs de connaissances complémentaires dans le développement de logiciels de CAO (conception assistée par ordinateur) pour la réalisation de systèmes intégrés dans les puces électroniques et la simulation de circuits imprimés. Citons l'exemple d'une start up qui réalise la conception et commercialisation de fonctions microélectroniques destinées aux circuits intégrés de plates formes sécurisées. Elle est positionnée sur l'innovation dans « les interfaces de communications à hautes vitesses » du système microélectronique. Ses connaissances s'intègrent donc dans un ensemble modulaire plus large (plate-formes sécurisées) et ses clients sont des fabricants de circuits intégrés pour objets communicants sécurisés. Des PME de ce profil sont associées dans des projets de R\&D principalement autour de la plate-forme technologique de conception gérée par l'association ARCSIS. Elles sont donc fournisseurs d'entreprises qui intègrent les technologies de la puce et se situent sur des marchés internationaux.

Dans un deuxième groupe, plus distant du cœur du paradigme dominant, se trouvent des PME aux briques de connaissances complémentaires à la microélectronique ou à la téléphonie mobile. Une partie de ces PME se situe dans le renouvellement des matériaux et logiciels liés au processus d'identification et de fonctionnalités de la carte à puce (intégration sur support souple) et dans le renouvellement même des produits permettant l'identification (tags, RFID ${ }^{16}$ et plus généralement des processus liés à l'identification et la sécurité des objets de confiance). Ces entreprises se sont portées candidates pour utiliser la plate-forme de micropackaging ${ }^{17}$ qui préexistait au pôle. L'autre partie de ces PME se situe à l'interface entre

\footnotetext{
${ }^{15}$ Statistique fournie par le pôle SCS, recensement de 2006 à Juin 2008. Définition de la PME selon la norme européenne (effectifs inf. à 250 salariés).

${ }^{16}$ En français, cela se traduit par «identification par radio fréquence »

${ }^{17}$ Il s'agit sur cette plate-forme de tester la sécurité de cartes à puces et autres composants, et de travailler à une intégration fiable des éléments électroniques sur différents supports.
} 
les équipementiers (fabrication d'objets portables communicants) et les opérateurs de télécommunications. Il s'agit d'experts en développement de logiciels embarqués de types «middleware » pour le multimédia mobile. Ces entreprises se sont organisées simultanément à la constitution du pôle autour d'une plate-forme technologique de test de logiciel pour la téléphonie mobile qui est financée par la Région. Parmi ces deux groupes de PME, certaines vont se positionner comme intégrateurs de technologies liées aux objets portables communicants et sécurisés ou comme intégrateurs de technologies liées à la mobilité, la vision, la transmission d'informations (données, images). Dans ces dernières entreprises, le développement logiciel ne représente plus qu'une brique dans les connaissances technologiques mises en œuvre. L'objet produit et les services associés sont, par conséquent, plus fongibles dans l'économie. Les clients potentiels sont très divers. Elles intègrent des connaissances d'autres PME dans le cadre de projet $R \& D$ du pôle renouvelant les configurations de réseaux du pôle.

\section{Figure 2 : Le positionnement des PME du logiciel et du multimédia dans le Pôle}

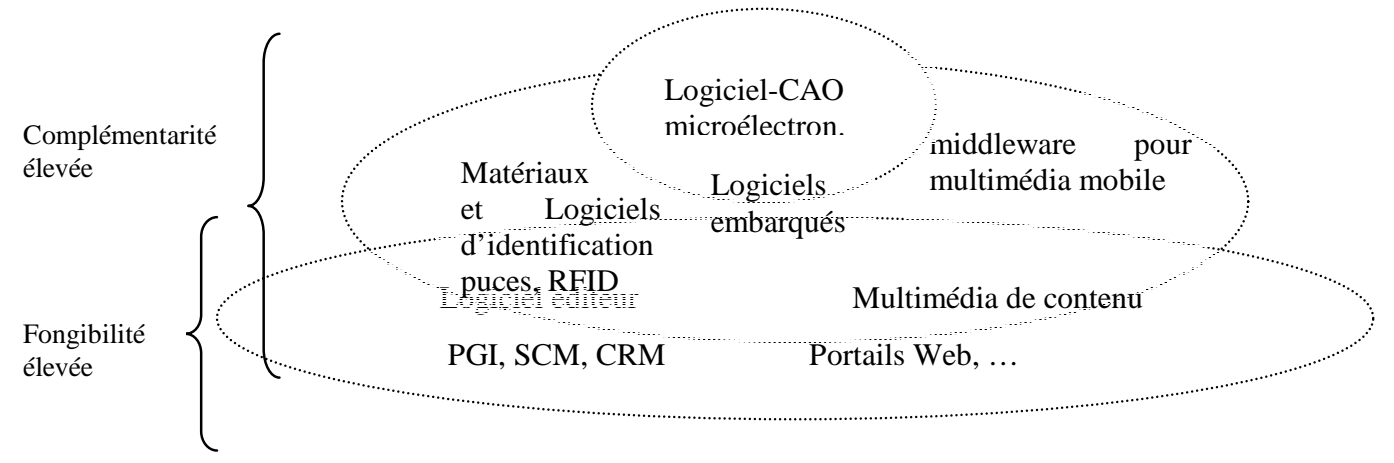

Enfin dans un troisième groupe se situent des entreprises du multimédia et du logiciel éditeur dont les connaissances s'adressent à tous types d'entreprises et d'organisation. Il s'agit de connaissances dans les domaines des services et produits de la communication, du marketing, du ludo-éducatif et du développement de systèmes d'information ${ }^{18}$. Si ces entreprises sont conscientes de l'enjeu lié aux savoirs sur le logiciel embarqué et le logiciel dédié aux objets portables pour être crédibles dans le cadre des projets R\&D du pôle, la distance semble trop grande en terme de corpus de connaissances spécifiques et de réseaux industriels. Par conséquent, leur savoir ne peut être directement articulé aux savoirs des acteurs présents dans les commissions thématiques du pôle puisqu'il concerne les fonctions de supports de gestion et d'administration (de la conception, des études, de la production) ainsi que les fonctions de communication et d'information. Ajoutons que la plupart d'entre elles restent en même temps focalisées sur l'objectif de croissance de leur activité de développement par l'accès aux grands comptes, et n'éprouvent pas, pour l'instant, la nécessité d'un détour par des projets R\&D collaboratifs. Les réponses à des appels d'offres sont une opportunité directe de développements nouveaux et innovants, lorsque ces PME détiennent les savoirs et langages pertinents de conception et développement; cela est le cas pour une grande majorité d'entre elles.

En résumé, ces entreprises aux savoirs les plus fongibles s'adressent à un large spectre d'entreprises plutôt qu'à des producteurs de technologies à l'intérieur du pôle. Dans la mesure où leur offre technologique est diffuse et ne s'identifie pas dans une stricte complémentarité

\footnotetext{
${ }^{18}$ De type progiciel de gestion intégré, supply chain management (SCM) ou bien customer relationship management (CRM) comme briques de ces systèmes d'information.
} 
vis-à-vis des entreprises innovantes du pôle, leur coordination avec ces dernières reste délicate du point de vue de la gouvernance du pôle. Ainsi se pose la question de l'articulation entre l'espace d'intervention du pôle et celui de la gouvernance territoriale en vue de favoriser la compétitivité de ces entreprises dans une perspective de développement de la filière TIC à l'échelle régionale.

\subsection{L'espace industriel du pôle comme lieu d'articulation d'une politique de soutien à l'innovation et de renforcement de filière}

La stratégie en matière d'innovation adoptée dans le cadre de la gouvernance territoriale a abouti à la mise en œuvre d'une politique industrielle territoriale visant à l'émergence de clusters d'innovation régionaux (les PRIDES ${ }^{19}$ ). Celle-ci vise à faciliter la croissance et l'amélioration des performances des PME territoriales, cibles prioritaires du dispositif, à partir de 5 leviers de développement complémentaires (l'innovation, la gestion des compétences et l'emploi, les TIC, l'international, le développement durable).

Dans le cadre de ce dispositif régional, le projet de mise en place d'une plate-forme multimédia vise à la mise sur le marché de produits ou services innovants, à l'image de ce qui se pratique déjà dans la filière régionale microélectronique (avec la plate-forme Cim Paca). L'enjeu en terme d'innovation pour les entreprises du multimédia est d'arriver à mettre sur le marché des produits ou des services qui sont exploitables sur l'ensemble de ces canaux de distribution (Internet, mobile, IP TV ...). Face à la multiplication des supports de diffusion, les produits et services associés subissent un cycle de vie très court et demandent à être réévalués en permanence. Dans ce contexte, une telle plate-forme est vue comme un outil de «laboratoire industriel» aidant à la mise au point de nouveaux développements technologiques de pointe mais aussi comme un moyen de tester de nouveaux services innovants en termes d'usage et de marché. Il peut permettre à terme un déplacement de ces PME vers des logiques collectives d'innovation plus poussées. La structure du pôle a joué un rôle de médiateur et de co-évaluateur de ce projet en interaction avec les évaluateursfinanceurs de la Région.

Du côté de l'association du logiciel éditeur, composé de compétences de développement traditionnelles et nouvelles fondées sur les langages de l'Internet, la logique d'action va plutôt s'orienter sur des démarches de marketing territorial et d'accès aux marchés grands comptes.

Un plan d'action est consigné dans une convention avec la Région et bénéficie d'une partie de l'enveloppe dédiée à l'animation des PRIDES dans le pôle SCS. Trois principaux projets vont être mis en œuvre entre 2007 et 2008. Un premier projet porte sur la constitution d'une carte d'identité de la filière (nombre de salariés selon les activités, croissance de l'emploi). La réalisation de cette étude permet aux acteurs principaux de l'association d'asseoir la légitimité des activités de l'association en montrant l'importance des PME et des TPE dans le secteur du développement logiciel ainsi que leur fragilité, impliquant des soutiens financiers publics. Audelà de cet aspect, l'étude permet aux éditeurs logiciels de mieux se situer dans leur filière et dans l'économie régionale et, en même temps, d'être plus visibles collectivement à l'extérieur, à partir de la mise à disposition de cette information sur le Web. Un deuxième projet vise à l'identification des compétences dans la filière logiciel et est supposé permettre une GPEC au niveau du cluster. Il s'agit d'une approche plus qualitative qui consiste à demander à chaque entreprise adhérente de l'association de définir, si elle le souhaite, ses compétences managériales, techniques et relationnelles. Cette information est présentée sur le Web via un outil de gestion des connaissances au niveau du territoire (dispositif KMP issu de la R\&D d'une unité mixte en économie-droit-gestion et de l'INRIA). Les responsables de

\footnotetext{
${ }^{19}$ Pôles Régionaux d'Innovation Economiques et Solidaires mis en place par la Région PACA.
} 
l'association attendent de cet outil une aide à la gouvernance des entreprises du logiciel éditeur. Un troisième projet subventionné par la DRIRE, porte sur le montage d'un GIE pour favoriser les réponses intégrées à des appels d'offres de grands comptes. On observe à partir des retours sur les réponses à appel d'offre que ces petites entreprises ne sont pas considérées comme crédibles, bien que leurs compétences intéressent les clients grands comptes, en raison de leur taille. Ce, en général au profit de majors qui n'ont pas forcément les solutions les plus adaptées et les moins coûteuses.

\section{Gouvernance territoriale et construction d'un espace légitime d'intervention du pôle.}

L'intégration industrielle des PME dans le pôle se doit d'être appréhendée au travers d'une conception large de l'innovation et de la compétitivité, au-delà de la seule dimension de R\&D. Partant de là, deux types de questionnements nous paraissent importants d'être posés. Tout d'abord, se pose la question délicate de l'articulation entre les différents acteurs de la gouvernance de l'innovation à l'échelle d'un territoire. Leur harmonisation ne va pas de soi et requiert l'émergence de nouvelles règles issues d'apprentissages collectifs. Nous discutons respectivement ces deux points en nous centrant sur le cas des PME du logiciel éditeur et du multimédia de contenu.

\subsection{Quelle articulation entre les différentes sphères de gouvernance à l'échelle territoriale?}

L'implication optimale des PME dans les projets collaboratifs d'innovation constitue un enjeu primordial pour un pôle de compétitivité. Toutefois, cet enjeu peut devenir au regard des caractéristiques de certaines populations de PME adhérentes un défi difficilement atteignable pour les raisons évoquées dans la section précédente. Outre le faible degré de proximité technologique qui constitue un frein indéniable, l'innovation n'est pas, pour la PME, le seul et unique levier de sa compétitivité. Cela pose la question de la frontière de l'espace légitime d'intervention d'un pôle de compétitivité. En effet, si le soutien global aux PME fait aussi partie des missions du pôle, l'expérience montre qu'il peut s'avérer difficile, au moins au départ, d'identifier très précisément les actions à mettre en place, les responsables du pôle ne bénéficiant pas nécessairement de l'expertise nécessaire en la matière. Cela peut alors se traduire par un désengagement de certaines PME qui ne trouvent pas satisfaction par rapport à leurs attentes.

Notre hypothèse est la suivante : c'est seulement par des apprentissages collectifs, c'est-à-dire par une évolution des règles communes ${ }^{20}$, et en partenariat avec les acteurs du territoire, qu'un pôle de compétitivité peut arriver à proposer un ensemble de services susceptibles de favoriser la compétitivité globale des PME sur le territoire. Eu égard à cette question, le pôle SCS nous semble constituer un cas tout à fait intéressant de négociations avec les autres acteurs territoriaux et, en particulier, avec l'institution politique du Conseil Régional qui a joué un rôle clé dans la structuration de la filière des TI.

Rappelons qu'un pôle de compétitivité doit remplir deux grandes missions: il doit tout d'abord favoriser une dynamique d'innovation collaborative performante susceptible de renforcer la compétitivité nationale voire internationale de certains tissus industriels. Il doit ensuite contribuer au développement territorial en favorisant la création d'emplois et d'entreprises technologiques. Dans un pôle mondial comme le pôle SCS, il existe de fait une

\footnotetext{
${ }^{20}$ Nous nous référons ici au cadre théorique de la régulation sociale tel qu'il est posé par J. D. Reynaud (1997), ainsi qu'aux travaux de Jacques de Maillard qui a su mettre en évidence ces logiques d'apprentissages collectifs dans le cadre de la transformation de la politique des villes et des banlieues (Maillard 2004).
} 
hiérarchisation implicite entre ces deux grandes missions. En effet, s'il veut être performant en matière d'innovation, il doit concentrer tous ses efforts sur cet objectif d'autant que ce type de pôle est généralement plongé, via les marchés de ses grandes entreprises, dans un univers de concurrence internationale acérée. L'objectif de développement territorial devient ainsi de fait secondaire. D'ailleurs à cet égard, le pôle SCS souligne explicitement dans sa communication externe qu'il participe à la création d'un écosystème approprié au développement régional mais qu'il n'en est nullement le seul instigateur.

De son côté, le Conseil Régional a adopté progressivement un positionnement stratégique complémentaire aux missions du pôle. Cette institution s'est en quelque sorte «professionnalisée » dans l'exercice de son mandat en matière de développement économique territorial. La loi de 2004 qui confère aux Conseils Régionaux un rôle de chef d'orchestre au niveau territorial a fortement contribué à cela. C'est dans le cadre de l'élaboration de son Schéma Régional de Développement Economique (2005-2006) que cette institution politique a initié, sur la base d'une concertation avec l'ensemble de ses partenaires territoriaux, une nouvelle forme de gouvernance que nous appelons à dessein une gouvernance territoriale.

$\mathrm{Au}$ premier abord, cette politique a suscité une certaine perplexité pour les acteurs économiques concernés qui n'arrivaient plus véritablement à discerner les missions respectives du Pôle de compétitivité et du Conseil Régional en matière d'innovation. Toutefois, pour ce dernier, la politique des PRIDES ne venait en rien concurrencer la politique d'innovation des pôles. Si elle partage, avec leur philosophie, l'idée que le management de l'innovation passe par une phase de coopération et une mise en réseau des acteurs, elle s'en éloigne en ce qu'elle s'appuie sur une vision plus globale de la compétitivité et de l'innovation des PME.

Pour le Conseil Régional, cette politique d'innovation, pensée comme un outil de développement territorial dédié à la compétitivité des filières industrielles, est vouée à être articulée au dispositif des pôles dans une perspective de gouvernance territoriale. Elle a en effet confié à la gouvernance du pôle SCS une mission de coordination de cette politique en partenariat avec les associations professionnelles. Ainsi, les associations Medmultimed et Médinsoft, vont émarger, à l'instar des autres associations du pôle de compétitivité, à ce dispositif régional.

\subsection{Le rôle des apprentissages collectifs dans l'émergence d'un système territorial d'innovation}

Le dispositif régional de soutien financier aux projets collectifs innovants entendu au sens large (définition d'Oslo) comporte deux volets complémentaires : le premier volet consiste à soutenir des actions collectives de filières économiques territoriales dans le but d'accroître leur ancrage territorial, et le second consiste à soutenir l'innovation en terme de produits et services en ciblant plus spécifiquement des projets de R\&D portés par des PME régionales. L'étude de l'appropriation de ces nouveaux dispositifs de financement de la Région coordonnés par le pôle est révélatrice des problématiques qui préoccupent le plus ces PME et des logiques d'apprentissages collectifs qui vont émerger.

L'étude du cas montre, en premier lieu, qu'elles se sont fédérées plus facilement et massivement autour d'actions collectives plutôt que de projets R\&D à leur dimension. Néanmoins, on perçoit une évolution dans leur positionnement vis-à-vis de projets collaboratifs innovants en terme de produits et services. Nous montrons dans l'analyse qui suit que ce double déplacement est lié à des apprentissages collectifs réalisés dans le cadre de l'élargissement de l'espace d'intervention et de coordination du pôle (Gadille, 2008). 
L'observation des actions collectives qui émergent des deux associations représentatives de ces PME du multimédia et du logiciel éditeur, révèle que celles-ci sont centrées sur une recherche de développement de la compétitivité globale de la filière. Néanmoins les logiques d'action se différencient en fonction des spécificités technologiques de ces activités. Du côté de l'association du multimédia, les demandes de financement vont porter, d'une part, sur la mise en place d'une coordination et coopération liées aux questions de formation et, d'autre part, sur la mutualisation d'équipements technologiques coûteux (plate-forme multimédia). Dans le domaine de la formation, il s'agit de réunir les entreprises du multimédia et les instituts de formation qui collaboraient peu jusqu'alors, avec pour objectif d'améliorer la relation entre l'offre et la demande sur le marché du travail local. Ces entreprises, jeunes en général, connaissent mal le marché de la formation; ce qui s'explique en partie par une profusion d'offres et un manque de visibilité sur les compétences délivrées par chacun des diplômes existants sur le périmètre régional. Il a été proposé de réfléchir en commun à la mise en place d'un référentiel de compétences métiers dans ce domaine afin d'établir une sorte de «langage commun» favorisant les interactions. Le pôle va seulement jouer un rôle d'observateur dans la négociation entre l'association et la Région. Cependant la connaissance de ce projet vient renforcer la légitimité d'une logique d'action dans le pôle en faveur de la question de la régulation de la formation et du marché du travail dans les filières qu'il coordonne. Une action plus générale sur les offres de formation dans l'ensemble des filières du pôle sera conduite avec un léger différé.

Du côté de l'émergence de projets de recherche finalisés financés par la Région, (appel à projets lancé fin 2006), même si l'on ne possède pas tout le recul pour estimer la capacité de participation dans ce domaine, on note une très faible participation. Il n'y a pour l'instant aucun projet issu du multimédia de contenu et seulement deux projets sont issus de développeurs de logiciel dans le domaine des systèmes d'information de type «aide à la décision pour les organisations collégiales » et «solutions e-business adaptées aux PME». Ces leaders de projet parviennent à nouer de nouveaux partenariats avec des industriels clients et des chercheurs de sciences sociales ou de l'ingénieur. Mais si ces projets ne sortent pas du cadre thématique défini par le pôle dans le sens où ils traitent des usages de TIC, ils en sortent en matière de légitimité vis-à-vis du cœur du paradigme dominant élargi à la téléphonie mobile et la traçabilité, fiabilité des objets communicants. On observe d'ailleurs que ces projets n'ont été labellisés qu'au terme de négociations serrées de la structure de gouvernance du pôle avec les experts évaluateurs issus du cœur du paradigme et de son premier cercle (microélectronique, téléphonie mobile, technologies d'identification). Pourtant ils s'inscrivent dans le cadre d'un développement territorial de la filière TIC sur la base d'une diffusion de nouvelles technologies qui impacteront la performance des autres PME.

Pour expliquer ce fait, nous revenons à la notion de connaissances et de ce qui fait sens à l'intérieur d'un paradigme technologique, au-delà des aspects de rivalité ou concurrence. Cette négociation pour résoudre le conflit crée un précédent et marque un déplacement dans l'établissement de ce qui fait référence dans l'évaluation des projets de R\&D. Notons qu'elle a été menée par l'acteur individuel en charge de l'ingénierie des projets qui ne provenait pas, à la différence du directeur général et de son adjoint, du cœur du paradigme dominant. On observe donc des logiques d'apprentissages collectifs (Maillard, 2004) à partir de l'intégration de nouvelles compétences au niveau organisationnel et l'établissement de nouveaux compromis au niveau de la gouvernance territoriale. Ces apprentissages sont corrélatifs de l'émergence de nouvelles règles dans le sens de l'élargissement de l'espace d'intervention légitime du pôle dans le tissu économique de la région. Ces observations suggèrent que l'on ne peut affirmer à l'instar de certains auteurs (Duranton et al., 2008) que la politique des pôles d'initiative nationale est uniquement un levier pour augmenter le niveau de concentration géographique. Elle est aussi ce que les régions en font dans le jeu d'une gouvernance 
territoriale qui peut se l'approprier et favoriser une levée des obstacles auxquels se heurte la croissance d'un cluster.

\section{Conclusion}

Le dispositif de pôle de compétitivité a été créé par l'Etat au niveau central et de façon indépendante vis-à-vis des Conseils Régionaux qui voient cependant leur légitimité grandir notamment en terme de développement économique. Ce nouveau dispositif incarne une politique industrielle de soutien à l'innovation centrée sur la $R \& D$ mais s'inscrit en région dans le cadre d'une gouvernance territoriale. Cette politique doit répondre à un double défi : celui de l'intégration optimale de PME innovantes non nécessairement centrées sur la R\&D et celui de la coordination entre la structure de gouvernance du pôle et les autres acteurs du territoire déjà présents et légitimes dans le domaine du développement économique (pôle de compétitivité, conseil régional, chambre de commerce et d'industrie, ...). Dans de nombreuses régions, des tensions ont alors émergé entre ces différents acteurs. En effet, le dispositif de pôle était tantôt perçu par certains comme une politique d'innovation territoriale efficace et tantôt, par d'autres, comme une couche supplémentaire de prise de décision dans laquelle ils se trouvent encastrés et/ou avec laquelle ils se trouvent en concurrence dans la définition des contenus, des modes et des espaces d'intervention légitimes.

Dans ce contexte, nous avons cherché à comprendre pourquoi, au-delà des aspects de gestion de la propriété intellectuelle et de la confiance, certaines entreprises représentées par leur association en conseil d'administration n'étaient pas impliqués dans les projets de R\&D. Nous avons ainsi mis l'accent sur l'importance de la nature des connaissances plus ou moins fongibles ou complémentaires vis-à-vis du cœur de paradigme et des systèmes technologiques préexistant à la création du pôle. Partant ensuite du fait que les PME à la marge de ce dispositif représentent un enjeu de développement économique pour la Région, nous avons montré comment celle-ci réussit à concilier sa propre logique d'action publique dans le champ économique avec celle du dispositif national de pôle axée sur la R\&D. Cela s'est traduit en particulier par un élargissement de l'espace légitime d'intervention et de coordination du pôle. Le compromis à la source de cet élargissement favorise des économies de transaction car le pôle se voit confier un rôle d'évaluateur et de conseiller auprès des différentes populations de PME qui n'ont pas une activité de R\&D au sens technologique du terme. Nous montrons qu'il permet surtout des apprentissages collectifs croisés entre différentes communautés professionnelles. On constate, en effet, une évolution du contenu des actions collectives dans le tissu des PME et simultanément, un déplacement des référentiels d'évaluation des projets R\&D dans le pôle. Ces déplacements restent fragiles mais ne peuvent être ignorés dans une perspective de compétitivité à long terme des tissus régionaux.

\section{Bibliographie}

C. Antonelli (2003), «Knowledge complementarity and fungeability : implication for regional stragtegy », Regional Studies, vol 37, N 6/7, p. 595-606

B. Carlsson (1994), «Technological Systems and Economic Performance », in The Handbook of Industrial Innovation, M. Dogson and R. Rothwell, Edward Elgar (Ed), Aldershot, p.13-24

B. Carlsson, R. Stankiewicz (1991), «On the nature, function and composition of technological systems », Journal of evolutionary economics, Vol.1, $\mathrm{N}^{\circ} 2$, p.93-118

G. Dosi (1982), «Technological paradigms and technological trajectories », Research Policy, $\mathrm{N}^{\circ} 11$, p. $147-162$. 
L. Dibiaggio et M. Ferrary (2003), «Communautés de pratique et réseaux sociaux dans la dynamique de fonctionnement des clusters de hautes technologies ", Revue D'économie Industrielle, $\mathrm{N}^{\circ} 103, \mathrm{p} .111-130$

G. Duranton, P. Martin et F. Mayneris (2008), Les pôles de compétitivité. Que peut-on en attendre?, ed. Rue d'ULM/ENS, Paris

S. Ehlinger, V. Perret et D. Chabaud (2007), «Quelle gouvernance pour les réseaux territorialisés d'organisations ? », Revue Française de Gestion, №170, p. 155-171

M. Gadille (2008), «Le tiers comme agent de réflexivité et accélérateur d'apprentissage collectifs : le cas du dispositif des pôles de compétitivité », Humanisme et Entreprise, $\mathrm{N}^{\circ} 289$, octobre, p. 61-79

J. Garnier (2008), Des anciens tissus productifs aux nouveaux clusters : quelle transition?, ouvrage collectif sous la direction de J. Garnier, l'Harmattan, Paris

J. Garnier et C. Lanciano-Morandat (2008), «Les origines du pôle », dans A. Mendez (dir), Quelle articulation entre les pôles de compétitivité et les tissus productifs locaux? Une mise en perspective de quatre pôles en Provence Alpes Côte d'Azur, rapport de recherche du LEST, Aix-en-Provence.

J.P. Gilly et J. Perrat (2004), « La dynamique institutionnelle des territoires entre gouvernance locale et régulation globale», dans Gouvernance locale et développement territorial : le cas des pays du Sud, A. Feruène (ed), Harmattan, Paris, p. 77-91.

C. Lanciano-Morandat et H. Nohara (2008), «Dynamique science industrie, plate-formes technologiques et projets collaboratifs: contribution à la construction d'un espace intermédiaire », A. Mendez (dir), dans Quelle articulation entre les pôles de compétitivité et les tissus productifs locaux? Une mise en perspective de quatre pôles en Provence Alpes Côte d'Azur, rapport de recherche du LEST, Aix-en-Provence

Ch. Longhi et J. Spindler (1999), Le développement local, Paris, librairie générale du droit et de la jurisprudence

A. Maillard (de), Réformer l'action publique, la politique de la ville et les banlieues, L.G.D.J. Droit et société, Paris

K. Pavitt (1984), «Sectoral patterns of techical change : toward a taxonomy and a theory » Research Policy, Vol. 13, p. 343-373

B. Pecqueur (2004), «Territoire et gouvernance: quel outil pertinent pour le développement? »dans Gouvernance locale et développement territorial : le cas des pays du Sud, A. Ferguène (ed), l'Harmattan, Paris, p. 27-47

M. Pélissier (2008), «Etude sur l'origine et les fondements de l'intelligence territoriale », dans Intelligence territoriale : l'intelligence économique appliquée au territoire, L. François (Coord), Lavoisier, Paris, p. 25-38

J.D. Reynaud (1997), Les règles du jeu. L'action collective et la régulation sociale, $3^{\text {ème }}$ édition, Armand Colin, Paris

J.B. Zimmerman (1998), L'émergence d'un tissu microélectronique dans les Bouches du Rhône, rapport de synthèse, étude financée par la Conseil Général des Bouches du Rhône. 